\title{
Hypertrichotic osteochondrodysplasia, Cantu type
}

INSERM

\section{Source}

INSERM. (1999). Orphanet: an online rare disease and orphan drug data base.

Hypertrichotic osteochondrodysplasia, Cantu type. ORPHA:1517

Cantu syndrome is a rare disorder characterized by congenital hypertrichosis, osteochondrodysplasia, cardiomegaly, and dysmorphism. 